\title{
Semi-diurnal and diurnal variation of errors in GPS precipitable water vapor at Tsukuba, Japan caused by site displacement due to ocean tidal loading
}

\author{
Yoshinori Shoji ${ }^{1}$, Hajime Nakamura ${ }^{1}$, Kazumasa Aonashi ${ }^{1}$, Akinori Ichiki ${ }^{1}$, Hiromu Seko ${ }^{1}$, \\ and Members of GPS/MET Japan Summer Campaign 1997 in Tsukuba \\ ${ }^{1}$ Meteorological Research Institute, 1-1 Nagamine, Tsukuba, Ibaraki 305-0052, Japan
}

(Received January 7, 2000; Revised September 9, 2000; Accepted September 9, 2000)

\begin{abstract}
Simultaneous GPS and water vapor radiometer (WVR) observations were carried out at Tsukuba, Japan from May 1 to June 30, 1998. The precise point positioning method of the GIPSY/OASIS-II software package (GIPSY) was used to retrieve precipitable water vapor (GPS_PWV) from GPS data, which was then compared with precipitable water vapor observed by WVR (WVR_PWV). They agreed quite well with the root mean square difference of less than $1.5 \mathrm{~mm}$. However, periodic variations were found in the difference between GPS_PWV and WVR_PWV (dPWV). It was also found that semi-diurnal or diurnal components of these variations had a positive correlation with site displacement due to Ocean Tidal Loading (OTL). Two months of dPWV data were decomposed by the period of a component of OTL, and then composite time series data with a period equal to that of the component were made. This process was performed for $\mathrm{K}_{1}, \mathrm{O}_{1}, \mathrm{M}_{2}$, and $\mathrm{S}_{1}$ components of OTL. In each component, a periodic variation in dPWV appeared which was similar to those of the simulated GPS_PWV errors from OTL effects calculated with 'GOTIC' (Sato and Hanada, 1984), a program for the computation of OTL effect. Inclusion of OTL effects into GIPSY analysis reduced dPWV. In the $\mathrm{M}_{2}$ component, the amplitude of the dPWV was reduced by about $80 \%$. This suggests that the OTL components calculated by the GOTIC succeeded in simulating the actual site displacement by OTL effects in Japan. On the other hand, in $\mathrm{K}_{1}$ components, the amplitude of dPWV without OTL in GIPSY is 1.5 times larger than the simulated GPS_PWV error, with considerable error remaining even in the case of GIPSY analysis with OTL. The error may be due to multi-path effect, temperature dependency on conversion from Zenith Wet Delay to PWV, or instrument dependency of WVR on temperature. Analysis utilizing much longer data periods than the present two months is required to overcome these difficulties.
\end{abstract}

\section{Introduction}

In Japan, the Geographical Survey Institute (hereafter referred to as GSI) has developed a large nation-wide GPS network consisting of about 1,000 observation sites. GSI routinely calculates the zenith total atmospheric delay (hereafter referred to as ZTD) every 3 hours from this network data. The accuracy of such delay data must be evaluated if they are to be used for meteorological purposes. As Japan is located in the Asian monsoon area, it is presumed that temporal and spatial variations of water vapor are more intense than in the temperate climate zone. In order to evaluate the accuracy of GPS estimated precipitable water vapor (hereafter referred to as GPS_PWV) and to diagnose its temporal variation, simultaneous observation of GPS and a water vapor radiometer (hereafter referred to as WVR) were conducted at the Meteorological Research Institute (MRI) in Tsukuba for 2 months from May 1 to June 30, 1998. The ZTD was estimated every 5 minutes using the precise point positioning method with GIPSY/OASIS-II software package (GIPSY) (Webb and Zumberge, 1993). GPS_PWV was then derived from ZTD with observed surface pressure and temperature. The GPS_PWV was compared to the WVR

Copy right(C) The Society of Geomagnetism and Earth, Planetary and Space Sciences (SGEPSS); The Seismological Society of Japan; The Volcanological Society of Japan; The Geodetic Society of Japan; The Japanese Society for Planetary Sciences. derived PWV (hereafter referred to as WVR_PWV). The root mean square (RMS) difference between the GPS_PWV and the WVR_PWV was less than $1.5 \mathrm{~mm}$ during the project period for no rain cases. This RMS difference is similar to those of other observational studies in Europe and U.S.A. (e.g., Duan et al., 1996), and is comparable to or less than the observational error of the PWV by radiosonde observation.

However, it was found that the difference between GPS_PWV and WVR_PWV (hereafter referred to as dPWV) sometimes increased, reaching 3-4 $\mathrm{mm}$. This large amplitude of dPWV constitutes a serious problem for meteorological application. At present, GSI determines only one solution of GPS site coordinates from one day data for continuous GPS analysis. However, in the future, when near real-time coordinate analysis with higher time resolution is introduced for earthquake prediction or crustal deformation analysis, 3-4 mm error of PWV, which means 6-7 cm error of the vertical coordinate, will constitute a crucial problem.

It was found that the temporal variation pattern of the dPWV with a large 3-4 mm amplitude can be classified into (1) semi-diurnal and diurnal oscillations and (2) shorter time variations of 2 to 3 hours. It was also found that the former strongly correlated with vertical site displacement due to ocean tidal loading (hereafter referred to as OTL) effect. In this paper, we will focus on the semi-diurnal and diurnal 
oscillations and describe the relationship between variation in $\mathrm{dPWV}$ and the OTL effect. We also show how well dPWV can be reduced by the current GPS analysis software (GIPSY) with an OTL model for the Japan Islands.

\section{Observation and Data Analysis \\ 2.1 GPS}

For the GPS observation, we set up an Ashtech Z-XII dualfrequency receiver with a choke-ring antenna covered by a cone-shaped radome. The GPS antenna was mounted on the top of a stainless pole, approximately $1.7 \mathrm{~m}$ in height, in the observation field at MRI. The receiver logged dualfrequency carrier-phase and pseudo-range measurements with a sampling interval of $10 \mathrm{~s}$. We attached a surface meteorological instrument to the GPS antenna pole, which observed surface pressure, temperature, and relative humidity every 1 minute.

The observation commenced on May 1, 1998. The GPS observation was interrupted from June 8 to 14 , resuming with another GPS receiver/antenna pair of the same type until June 30.

The GIPSY was used with a precise point positioning strategy to estimate site coordinates and ZTD. Ionospheric-free linear combination of phase data (LC) was formed. We used the IGS recommended value for antenna phase center offsets. The satellite clock collections, orbits, and earth-rotation parameters were provided by the Jet Propulsion Laboratory (JPL) of the National Aeronautics and Space Administration (NASA). The earth-rotation parameter and orbits were based on the International Terrestrial Reference Frame (ITRF)-96. We used a cut-off elevation angle of 15-degrees. The a priori sigma for LC measurement was $1 \mathrm{~cm}$, and no elevation dependent weight was assigned. The site position displacement due to solid Earth tide was modeled using the model of Williams (1970). GIPSY uses a Kalman filtering technique to model the ZTD and other time-dependent parameters. The ZTD was modeled as a random walk variable with process noise of $0.17 \mathrm{~mm} / \mathrm{s}^{1 / 2}$, which allows $1 \mathrm{~cm}^{2}$ variation of tropospheric delays after 1 hour. Niell's mapping function (Niell, 1996) was used to estimate ZTD. The tropospheric delay gradient was not estimated in this paper.

In Japan, the treatment of the OTL effect is crucial when modeling site position because the Japan Islands are surrounded by ocean. Spatial variation of site displacement due to OTL effect is very large because of its sensitivity to the distance from the ocean, depth of the sea and geographical features of the shore. For example, amplitudes of $\mathrm{M}_{2}$ component range from $5 \mathrm{~mm}$ to $2 \mathrm{~cm}$ within Japan (Sengoku and Sato, 1995). In GIPSY, the displacement of a site coordinate $\Delta Z_{j}(t)$ due to OTL at a station $j$ at arbitrary time $t$ can be given as the sum of the OTL components as follows:

$$
\Delta Z_{j}(t)=\sum_{i=1}^{N} A_{i}^{j} \cos \left(\omega_{i} t+V_{i}-\delta_{i}^{j}\right)
$$

where $\omega_{i}$ is the frequency of $i$-th tidal component, $V_{i}$ is the astronomical argument, and $A_{i}^{j}$ and $\delta_{i}^{j}$ are amplitudes and Greenwich phase lag, respectively. The amplitude and phase lag at our project site in Tsukuba was calculated with a program for OTL effect computation named "GOTIC" (Sato and Hanada, 1984). The GOTIC can evaluate the loading effects for 11 main tidal constituents by using Schwiderski's ocean tidal model (Schwiderski, 1981). The accuracy of topographic maps employed in GOTIC is about 0.5 minutes of arc for any place in Japan. The integral method is used to take into account the effect of the finite area of the meshes near the station. Table 1 gives $A_{i}^{j}$ and $\delta_{i}^{j}$ computed for the project site.

Since many ocean tidal components have periods close to 24 hours, we constructed 36-hour session data by combining 24-hour session data which overlapped each other by 6 hours in order to avoid the end effect of the session, and performed GPS analysis with the 36-hour session data instead of the 24-hour session data.

The first step of the GPS analysis was to determine site coordinates. Starting from $999 \mathrm{~m}$ constraint in $x, y$, and $z$ coordinates, we repeated the GPS analysis on the two months of observed data, tightening the constraint gradually $(100 \mathrm{~m}$, $10 \mathrm{~m}$, and $5 \mathrm{~m}$ ). As a result, the standard deviations of daily horizontal and vertical components of site coordinates reached about $5 \mathrm{~mm}$ and $10 \mathrm{~mm}$, respectively.

In the second step, we fixed the site coordinate to the average of the analyzed values during the 2-month observation period. To study the impact of OTL modeling in GIPSY on PWV estimation, we performed two analyses, one including the effect of OTL and the other without it. In both analyses, one session analysis estimated a 36-hour time sequence of ZTD with a 5-minute interval. Each 36-hour time sequence data overlapped other sequence data for the first and last 6 hours. For retrieving PWV from ZTD, we only used 24hour time sequence data in the middle of the 36-hour time sequence data of ZTD, excluding the first and last 6-hour time sequence data.

To calculate PWV from ZTD, Zenith Hydrostatic Delay (hereafter referred to as ZHD) was first calculated (Davis et al., 1985), using 5-minute averaged data of observed surface pressure. Zenith Wet Delay (hereafter referred to as ZWD) was then derived by subtracting ZHD from ZTD. Finally, PWV was estimated from ZWD using a conversion coefficient calculated from the surface temperature by means of the formula proposed by Bevis et al. (1992). The conversion coefficients ranged from 0.156 to 0.165 during the observation period.

\subsection{Water Vapor Radiometer}

We set up a 23.8 and $31.4 \mathrm{GHz}$ dual frequency WVR (Ra-

Table 1. Amplitudes and phase of 11 main tidal components of site displacement due to OTL at MRI (calculated by GOTIC).

\begin{tabular}{|c|c|c|c|c|c|c|c|}
\hline & U-D & $\mathrm{N}-\mathrm{S}$ & E-W & U-D & N-S & E-W & \multirow[b]{2}{*}{ period } \\
\hline & \multicolumn{3}{|c|}{ amplitude (mm) } & \multicolumn{3}{|c|}{ phase (degree) } & \\
\hline Ssa & 0.97 & 0.07 & 0.29 & 285.63 & 99.37 & 260.31 & $4382.911 \mathrm{~h}$ \\
\hline $\mathrm{Mm}$ & 0.49 & 0.08 & 0.08 & 223.99 & 24.91 & 208.02 & $661.309 \mathrm{~h}$ \\
\hline Mf & 0.17 & 0.04 & 0.04 & 170.52 & 237. & 148.08 & $327.859 \mathrm{~h}$ \\
\hline $\mathrm{Q}_{1}$ & 1.72 & 0.23 & 0.35 & 198.04 & -122.09 & -12.84 & $26 \mathrm{~h} 52 \mathrm{~m} 06.1 \mathrm{~s}$ \\
\hline $\mathrm{O}_{1}$ & 8.44 & 1.27 & 1.72 & 203.33 & -108.30 & -10.09 & $49 \mathrm{~m} 09.6 \mathrm{~s}$ \\
\hline $\mathrm{P}_{1}$ & 3.33 & 0.52 & 0.69 & -137.58 & -86.95 & 9.82 & $24 \mathrm{~h} 03 \mathrm{~m} 57.2 \mathrm{~s}$ \\
\hline $\mathrm{K}_{1}$ & 10.77 & 1.72 & 2.17 & -137.51 & -88.81 & 10.01 & $23 \mathrm{~h} 56 \mathrm{~m} 04.1 \mathrm{~s}$ \\
\hline $\mathrm{N}_{2}$ & 1.25 & 0.36 & 0.37 & 69.77 & -91.63 & -200.69 & $12 \mathrm{~h} 39 \mathrm{~m} 30.1 \mathrm{~s}$ \\
\hline $\mathrm{M}_{2}$ & 8.27 & 1.91 & 3.01 & 55.14 & -83.21 & -187.22 & $12 \mathrm{~h} 25 \mathrm{~m} 14.2 \mathrm{~s}$ \\
\hline $\mathrm{S}_{2}$ & 4.25 & 0.76 & 1.40 & 77.74 & -67.14 & -146.80 & $12 \mathrm{~h} 00 \mathrm{~m} 00.0 \mathrm{~s}$ \\
\hline $\mathrm{K}_{2}$ & 1.20 & 0.24 & 0.38 & -279.08 & -61.60 & -145.07 & $11 \mathrm{~h} 58 \mathrm{~m} 02.0 \mathrm{~s}$ \\
\hline
\end{tabular}


diometric WVR 1100) near the GPS receiver. From May 1 to 5 , we observed microwave brightness temperature (TB) in the vertical direction only, but from 0800 UT May 6, it was observed not only in the vertical but also along 12 slant paths at azimuths of every 30 degrees with an elevation angle of every 20 degrees. We only used vertical direction data for the comparison with GPS_PWV and radiosonde observation. Sampling interval of the WVR_PWV was about every 1 minute for May 1-5, and about every 10 minutes for the rest of the project period. WVR_PWV are not reliable when a large amount of liquid water content (LWC) is retrieved with WVR. This is because predominant microwave radiation from raindrops masks the emission from water vapor. Therefore, we did not use the data when retrieved LWC exceeded $0.3 \mathrm{~kg} / \mathrm{m}^{2}$, considering that rain or high humidity conditions existed. There were no WVR observations for about $30 \%$ of the 2 -month project period. Since it is difficult to calibrate absolute TB values with a ground-based WVR, there arises an instrument specific bias in WVR observed TB value, which results in PWV bias. In our experiment, we compared GPS_PWV and WVR_PWV with the 12 hourly radiosonde measurement at the Aerological Observatory (Tateno) next to the MRI. WVR_PWV had a bias of about $4 \mathrm{~mm}$ against the radiosonde derived PWV, but the bias of GPS_PWV to the radiosonde PWV was only $0.4 \mathrm{~mm}$. So, we adjusted the bias of WVR_PWV to the radiosonde derived PWV to be zero.

\subsection{Estimation of GPS_PWV error from OTL}

Beutler et al. (1988) showed that a bias of $1 \mathrm{~mm}$ in ZTD causes a bias of about $2.9 \mathrm{~mm}$ in the vertical coordinate of a site for an elevation cut off angle of 20 degrees. This also implies that, if there is an error in the vertical coordinate due to neglect of the OTL effect, an error will result in ZTD. To confirm this relation for our case, we calculated the difference in the ZTDs analyzed with and without OTL. Then we compared it to the vertical component of site displacement calculated from 11 OTL components as stated in Subsection 2.1. Figure 1 shows that a good linear relationship exists between them. The magnitudes of ZTD difference were $27 \%$ of those of the vertical site displacement due to OTL. Hereafter, as a reference for error evaluation, we will

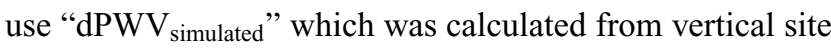
displacement due to 11 components of the OTL:

$$
\mathrm{dPWV}_{\text {simulated }}=\mathrm{UD} \times 0.27 \times \mathrm{PI}
$$

where UD is the vertical site displacement due to OTL effect, and PI is the conversion coefficient from ZWD to PWV as mentioned in Subsection 2.1.

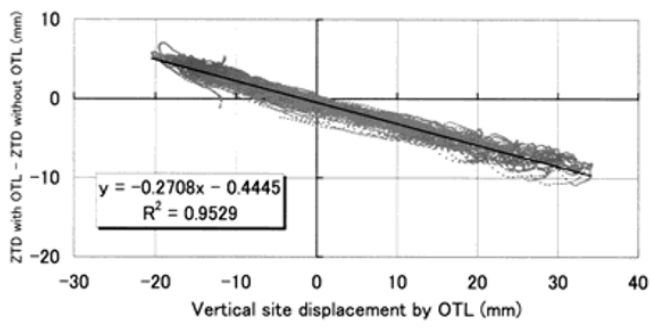

Fig. 1. A scatter diagram of difference in ZTDs (with OTL - without OTL) to vertical site displacement for project data from May 1 to June 30, 1998 at MRI. The straight black line represents the result of linear regression.

\section{Comparison} WVR PWV

between GPS_PWV

and

Figure 2 shows time sequences of GPS_PWV without OTL, WVR_PWV, and radiosonde derived PWV for the entire 2-month period of the project. It shows a good agreement among three PWVs as demonstrated by many other researchers (e.g., Duan et al., 1996; Ohtani and Naito, 2000). Although there were no WVR observations under rainy weather conditions, the agreement between GPS_PWV and radiosonde PWV was good. To evaluate the accuracy of GPS_PWV, we constructed a scatter diagram for WVR_PWV and GPS_PWV (not shown). We also calculated linear regression coefficients and bias removed RMS difference between GPS_PWV and WVR_PWV. The results are summarized in Table 2. It shows that 5-minute estimation of the GPS_PWV without OTL agreed well with WVR_PWV with RMS difference of $1.3 \mathrm{~mm}$. When the OTL is included in the analysis, a positive impact is observed reducing RMS difference to $1.1 \mathrm{~mm}$. Table 2 also shows the result when the coordinates were estimated in the process to determine ZTD with a constraint of $5 \mathrm{~m}$, which is often adopted in the GPS analysis. Obviously, the analysis of the fixed coordinates gave better estimations of PWV. When coordinates were not fixed, offset of ZTD and site coordinates occurred. In $5 \mathrm{~m}$ constrained cases, the slope of linear regression was smaller and the bias larger than in the fixed case. Therefore, for precise estimation of $\mathrm{PWV}$, it is preferable to perform
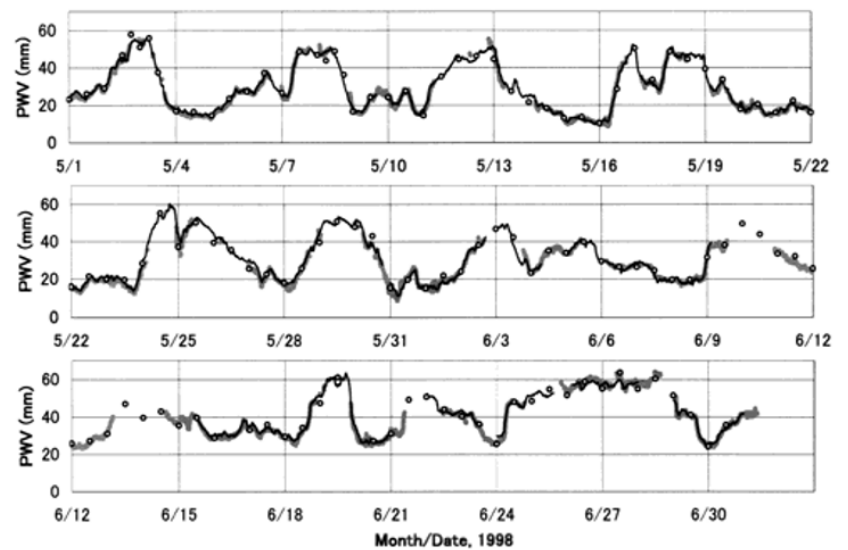

Fig. 2. PWVs at MRI from May 1 to June 30, 1998. Small circles are radiosonde PWV, thick gray lines are 5-minute averaged WVR_PWV, and thin black lines are 5-minute GPS_PWV (without OTL).

Table 2. Comparison of GPS_PWV to WVR_PWV during the project period from May 1 to June 30,1998. "slope of linear regression" and "y-intercept of linear regression" are tilt and y-intercept of a line resulting from fitting by linear regression, respectively.

\begin{tabular}{l|lcc}
\hline & & without OTL & with OTL \\
\hline \multirow{3}{*}{ coordinates } & slope of linear regression & 0.9732 & 0.9748 \\
fixed & y-intercept of linear regression & 1.3247 & 1.2006 \\
& GPS-WVR bias (mm) & 0.59 & 0.51 \\
& GPS-WVR bias removed rms $(\mathrm{mm})$ & 1.28 & 1.09 \\
\hline \multirow{3}{*}{ coordinates } & slope of linear regression & 0.9588 & 0.9595 \\
constrained & y-intercept of linear regression & 1.7511 & 1.6715 \\
$5 \mathrm{~m}$ & GPS-WVR bias (mm) & 0.62 & 0.56 \\
& GPS-WVR bias removed rms $(\mathrm{mm})$ & 1.32 & 1.14 \\
\hline
\end{tabular}




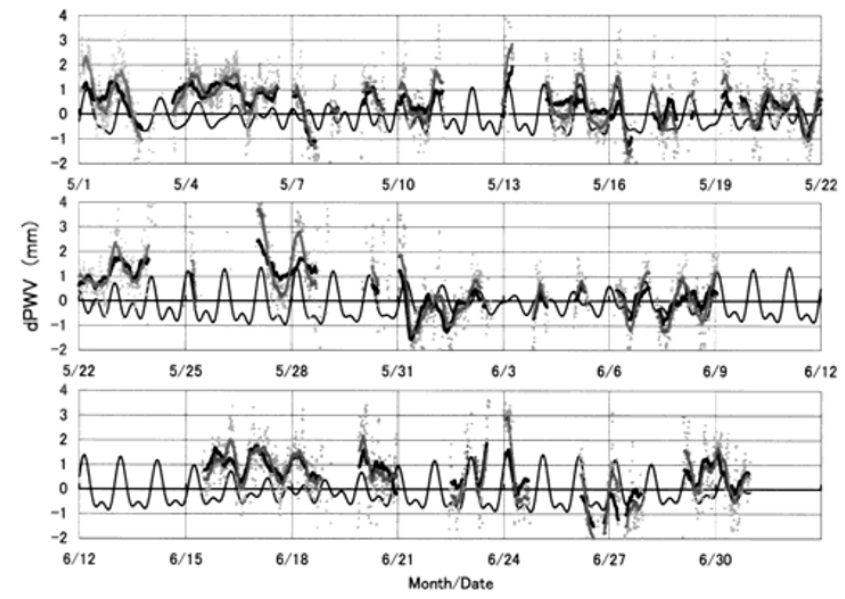

Fig. 3. Time sequences of dPWV from May 1 to June 30, 1998. Small gray dots are dPWV without OTL, thick gray lines are 6-hour running mean of dPWV without OTL, thick black lines are 6 hour running mean of dPWV with OTL, and thin black lines are 6 hour running mean of the $\mathrm{dPWV}_{\text {simulated }}$.

GPS analysis with the fixed coordinates, although the improvement is small compared to other errors such as OTL and multi-path effect.

Figure 3 shows the time sequence of the dPWV and the

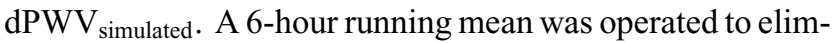
inate short time scale variations. Large differences between GPS_PWV and WVR_PWV with a period of equal to or less than one day were sometimes occurred. The dPWV without OTL (thick gray line) showed large temporal diurnal and/or semi-diurnal variation with the amplitude sometimes being as large as $3 \mathrm{~mm}$. The variation of $\mathrm{dPWV}$ without OTL

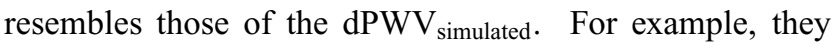
agreed quite well from May 15 to 17, from May 31 to June 2 , and from June 6 to 9 . From May 4 to 6 , from May 22 to 24, from May 27 to 29, and from June 16 to 19, tendencies were similar but there were biases between GPS_PWV and WVR_PWV. When the OTL effect is included in GIPSY analysis (thick black line), reduction of dPWV can be noted. For example, there was obvious improvement from May 15 to 17 and from May 27 to 29. However, improvement was small and the difference sometimes greater for other periods.

\section{Composite Time Sequence Data of dPWV with OTL Periods}

To study the relation between dPWV and the effects of each component of the OTL, we separate the two months of data into small time series data with a period of an OTL component and then average the small time series data to get a composite time series data with the OTL component period. For composition, we chose $\mathrm{K}_{1}, \mathrm{O}_{1}, \mathrm{M}_{2}$, and $\mathrm{S}_{1}$ components. $\mathrm{K}_{1}, \mathrm{O}_{1}$, and $\mathrm{M}_{2}$ have large vertical amplitudes. The vertical amplitude of the $\mathrm{S}_{1}$ component is small, but the component period is 24 hours. There can be other sources besides OTL, which cause 24-hour periodic variations in dPWV. For example, variation in atmospheric surface temperature from day to night may cause error in GPS_PWV during the process of converting ZTD to PWV (Subsection 2.1) as well as during the retrieval process of PWV in WVR.

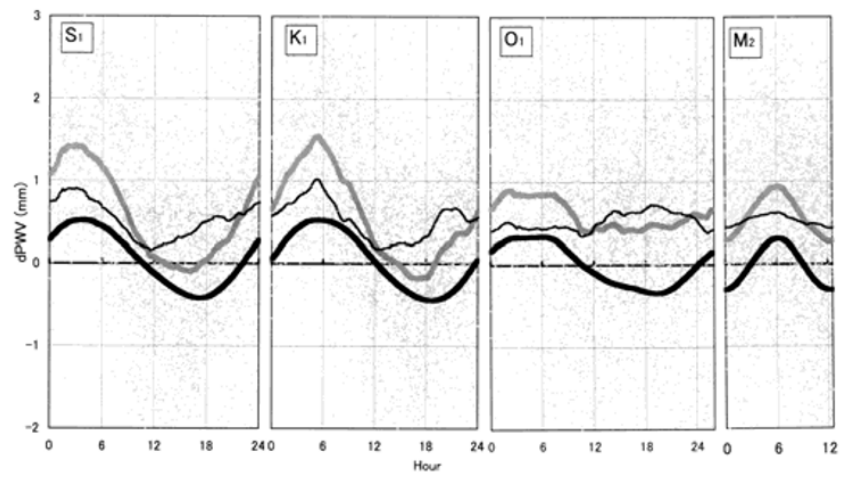

Fig. 4. Composite time series of dPWV and the dPWV $\mathrm{V}_{\text {simulated }}$ by $\mathrm{S}_{1}, \mathrm{~K}_{1}, \mathrm{O}_{1}$ , and $\mathrm{M}_{2}$ periods. Thick gray lines are composite time series of dPWV without OTL, thin black lines are composite time series of dPWV with OTL, and thick black lines are composite time series of $\mathrm{dPWV}_{\text {simulated }}$. Gray dots are dPWV without OTL before averaging.

By making composite time series data from 2 months of dPWV data, the effects of other tidal components may be cancelled. For example the $\mathrm{K}_{1}$ and $\mathrm{M}_{2}$ components have periods of 23 hours 56 minutes and 12 hours 25 minutes (24 hours 50 minutes in two periods), respectively. The difference is about 54 minutes. When we make a composite time series data with the $\mathrm{K}_{1}$ period, the error component with the $\mathrm{M}_{2}$ period will be almost canceled out, if the original data is longer than 27 days. On the other hand, the periods of $\mathrm{S}_{1}$ and $\mathrm{K}_{1}$ differ by only 4 minutes. Making composite data using 2 months of data is too short to completely separate the effect of these two components. Composite time series data were made for $\mathrm{dPWV}$ as well as the $\mathrm{dPWV}_{\text {simulated }}$ only when WVR_PWVs were obtained. Thus, $70 \%$ of the data were used.

Figure 4 shows composite time series data for $S_{1}, K_{1}$, $\mathrm{O}_{1}$, and $\mathrm{M}_{2}$ components. There are biases between the dPWV data and the $\mathrm{dPWV}_{\text {simulated }}$ as mentioned before (Section 3). The dPWV without OTL (thick gray lines) generally shows pure sinusoidal curves, which resemble those of the $\mathrm{dPWV}_{\text {simulated }}$ (solid black lines). The agreement of the $\mathrm{M}_{2}$ component is especially remarkable. The curves of other components have some high frequency irregularities. When the OTL was included in the GIPSY analysis, the amplitudes of the dPWV curves were reduced for all components (thin solid lines). As before, improvement of the $\mathrm{M}_{2}$ component is remarkable.

To discuss quantitatively, we fit a sinusoidal curve to composite data of dPWV, as follows,

$$
x(t) \fallingdotseq a_{0}+a_{1} \times \sin (\omega t-\phi)+\text { residual }
$$

where $\omega$ is the frequency of the corresponding OTL component, $a_{0}$ is the bias, $a_{1}$ and $\phi$ are amplitude and phase. We determined $a_{0}, a_{1}$, and $\phi$ by the least square method so that the residual will be minimal. Table 3 shows the results of the curve fitting for dPWV with and without OTL together with the $\mathrm{dPWV}_{\text {simulated. }}$.

In the $\mathrm{M}_{2}$ component, the amplitude and phase of a sinusoidal curve fitted to dPWV without OTL agrees well with

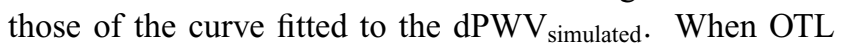
effect was included, the amplitude was reduced from 0.64 
Table 3. Summary of sine curve fitting to composite data of dPWV.

\begin{tabular}{llccc}
\hline & & & & \\
& & & dPWV & \\
simulated & $\begin{array}{c}\mathrm{dPWV} \\
\text { without OTL }\end{array}$ & $\begin{array}{c}\mathrm{dPWV} \\
\text { with OTL }\end{array}$ \\
\hline $\mathrm{S}_{1}$ & bias (mm) & 0.05 & 0.58 & 0.53 \\
& amplitude (mm) & 0.94 & 1.50 & 0.60 \\
& phase (deg) & -64.6 & -49.2 & -20.3 \\
& rms (mm) & 0.035 & 0.078 & 0.074 \\
\hline $\mathrm{K}_{1}$ & bias (mm) & 0.05 & 0.58 & 0.53 \\
& amplitude (mm) & 0.98 & 1.54 & 0.61 \\
& phase (deg) & -89.2 & -73.4 & -43.0 \\
& rms (mm) & 0.025 & 0.094 & 0.112 \\
\hline $\mathrm{O}_{1}$ & bias (mm) & 0.07 & 0.61 & 0.53 \\
& amplitude (mm) & 0.66 & 0.46 & 0.25 \\
& phase (deg) & -66.1 & -54.0 & 98.8 \\
& rms (mm) & 0.029 & 0.067 & 0.056 \\
\hline $\mathrm{M}_{2}$ & bias (mm) & -0.01 & 0.60 & 0.52 \\
& amplitude (mm) & 0.62 & 0.64 & 0.17 \\
& phase (deg) & -172.1 & -167.0 & -155.0 \\
& rms (mm) & 0.006 & 0.016 & 0.013 \\
\hline
\end{tabular}

$\mathrm{mm}$ to $0.17 \mathrm{~mm}$ (about $80 \%$ improvement).

In the $\mathrm{K}_{1}$ composition, the amplitude of $\mathrm{dPWV}$ without OTL is $1.54 \mathrm{~mm}, 1.5$ times larger than that of the $\mathrm{dPWV}_{\text {simulated. }}$ The amplitude was reduced by $0.93 \mathrm{~mm}$ with OTL, which is close to the amplitude of the $\mathrm{dPWV}_{\text {simulated }}$ $(0.98 \mathrm{~mm})$. Therefore, it may be said that inclusion of OTL effect worked well in reducing the errors in $\mathrm{K}_{1}$ component. However, there still remained $0.6 \mathrm{~mm}$ amplitude error and considerable phase difference. The period of the $\mathrm{K}_{1}$ component is the same as that of the GPS satellite orbit configuration. Composite data with $\mathrm{K}_{1}$ period may indicate the multi-path effect. Of course, this remaining error may be due to insufficient separation of other tidal components from $\mathrm{K}_{1}$ component, resulting from the use of only 2 months of data to produce the composite time series. Composite data of the period of $\mathrm{S}_{1}$ shows a similar pattern to that of $\mathrm{K}_{1}$ composition. The remaining error in $\mathrm{S}_{1}$ composite data with OTL may include the effect of diurnal variation of atmospheric temperature through the conversion from ZWD to PWV as well as the WVR analysis. However, because the periods of $\mathrm{S}_{1}$ and $\mathrm{K}_{1}$ are very close to each other, these two components were not separated well in this study. Therefore, it is difficult to discuss the relation between the error and the diurnal variation of temperature and/or other effects.

Compared with $\mathrm{M}_{2}, \mathrm{~K}_{1}$, and $\mathrm{S}_{1}$, the variation pattern of $\mathrm{O}_{1}$ composite data of dPWV without OTL is somewhat flat and differs from that of the $\mathrm{dPWV}_{\text {simulated. When OTL effect is }}$ included, the amplitude was reduced slightly, but the phase shifted about 190 degrees, almost being reversed. Since the period of $\mathrm{O}_{1}$ component differs considerably from other tidal components, it can be expected that the composite time series of the two-month data should separate the $\mathrm{O}_{1}$ component well from other components. This result of the $\mathrm{O}_{1}$ component may be attributed to a lack of about $30 \%$ of the WVR observation data, due to rainfall. The curve of the $\mathrm{dPWV}_{\text {simulated }}$ is not a pure sinusoidal one in Fig. 4. However, by making a composite time series of the $\mathrm{dPWV}_{\text {simulated }}$ using full 2 months data, we obtained a pure sinusoidal curve (not shown). This fact indicates that the missing period of WVR affected the composite process of $\mathrm{O}_{1}$ component.

\section{The OTL Effect on Double Difference Analysis}

We also tried the so-called "absolute method (Duan et al., 1996)" with the Bernese Ver. 4.0 software (Rothacher and Mervart, 1996), to study the OTL effect on double difference strategy. The LC combination of phase data was formed. Precise orbits, satellite clocks, earth-rotation parameters, and antenna phase center offsets from IGS were used. In the analysis, we used the GPS data above a 15-degree elevation angle with a sampling rate of 30 seconds. The mapping function available in Bernese Ver. 4.0 was cosine of zenith angle. To separate ZTDs at each station, seven baselines longer than $900 \mathrm{~km}$ were introduced to the analysis network (Fig. 5). The a priori sigma for L1 and L2 observation are $1.1 \mathrm{~mm}$, and no elevation dependent weight is assigned. In Bernese Ver. 4.0, the effect of solid earth tides is modeled according to the IERS Standards 1992 (McCarthy, 1992), but the OTL effect was not modeled. Therefore, we could only investigate cases without OTL. ZTD at MRI were estimated every hour by fixing coordinates of all sites in the analysis network to the 2-month averages. Then ZTD were converted to PWV as described in Subsection 2.1.

Figure 6 is a result of the composite time series of dPWV by $\mathrm{S}_{1}$ period. Compared with GIPSY's dPWV without OTL, the amplitude was almost the same but a different pattern was observed with a semi-diurnal variation.

According to Sengoku and Sato (1996), the amplitude of the site displacements in the vertical direction due to the OTL

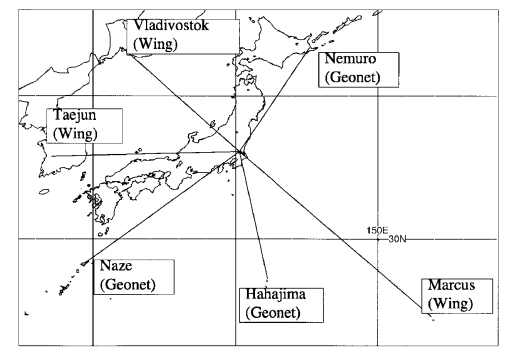

Fig. 5. GPS analysis network used in Bernese Ver. 4.0. GEONET is the operational GPS network site of the Geographical Survey Institute, and Wing is the Western Pacific Integrated Network of GPS.

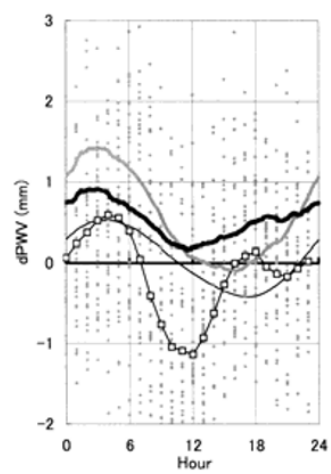

Fig. 6. Comparison of dPWV composite time series by $\mathrm{S}_{1}$ period. The thin black line with squares is the composite time series of Bernese dPWV, the thick gray line is the composite time series of GIPSY dPWV without OTL, thick black line is the composite time series of GIPSY dPWV with OTL, and black thin line is composite time series of $\mathrm{dPWV}_{\text {simulated }}$. The gray dots are Bernese dPWV (before averaging). 
is about $1 \sim 2 \mathrm{~cm}$ on average around Japan, and greater in the Nansei Shoto (islands located in the south-west of Japan) where vertical amplitude of the $\mathrm{M}_{2}$ component exceeds $2 \mathrm{~cm}$. In double-difference analysis, miss modeling of the site displacement of one site affects the solutions at other sites. This may be a reason why the dPWV by Bernese Ver. 4.0 shows a different pattern from those of GIPSY. In addition, there is a possibility that the composite process may not fully separate the $\mathrm{S}_{1}$ component from other components because of the data were calculated at intervals of 1 hour, as indicated by the existence of semi-diurnal variation in the composite data. Further detailed examination of this aspect is necessary.

\section{Conclusions}

We analyzed GPS data at MRI, Tsukuba from May 1 to June 30, 1998, with GIPSY's precise point positioning method and retrieved PWV (GPS_PWV) in 5-minute intervals, which were then compared with PWV derived by WVR (WVR_PWV). GPS_PWV agreed well with WVR_PWV with RMS difference of $1.3 \mathrm{~mm}$ without Ocean Tidal Loading (OTL). When the OTL was included in the analysis, a positive impact was observed reducing RMS difference to $1.1 \mathrm{~mm}$.

However, the difference between GPS_PWV and WVR_PWV (dPWV) without OTL showed diurnal and/or semi-diurnal variations with amplitudes sometimes as large as $3 \mathrm{~mm}$. In order to separate the contribution of each ocean tidal component to the $\mathrm{dPWV}$, we constructed composite time series data from the two months project data, which had periods of $\mathrm{K}_{1}, \mathrm{O}_{1}, \mathrm{M}_{2}$, and $\mathrm{S}_{1}$ components.

In $\mathrm{M}_{2}$ composite data, the pattern of $\mathrm{dPWV}$ without OTL was similar to that expected from site displacement due to the OTL model ( $\left.\mathrm{dPWV}_{\text {simulated }}\right)$. The dPWV with OTL showed about $80 \%$ reduction of the error amplitude. This suggests the program to calculate the OTL effect, GOTIC (Sato and Hanada, 1984), calculated the OTL components in Tsukuba well and the treatment of the OTL effects in GIPSY was adequate, at least for the $\mathrm{M}_{2}$ component. For the $\mathrm{K}_{1}$ composite data, the amplitude of dPWV without OTL was 1.5

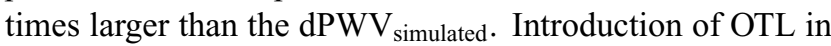
GIPSY reduced the amplitude by about the same magnitude

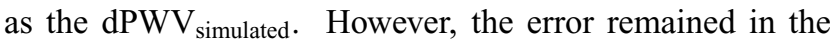
dPWV with OTL, which has $0.6 \mathrm{~mm}$ amplitude. This may be induced by multi-path effect or due to temperature dependency on the conversion from ZWD to PWV and on the retrieval method of WVR. Similar results were obtained for $\mathrm{S}_{1}$ composite data. We could not clarify the sources of these errors in this present composite time series data because two months is too short to separate components with different periods. We need much longer time periods of data, i.e. longer than 180 days, so that separation of the $\mathrm{K}_{1}$ component from other components, especially, $\mathrm{S}_{1}$, can be achieved. In $\mathrm{O}_{1}$ composite data, a missing period of WVR_PWV resulted in poor separation of the $\mathrm{O}_{1}$ component from other components. The dPWV with OTL reduced the amplitude but the phase reversed so that the correlation to the $\mathrm{dPWV}_{\text {simulated }}$ became worse.

We also tested the effect of OTL on double difference anal- ysis using Bernese Ver. 4.0, which did not include the OTL model. Composite data of the dPWV by Bernese Ver. 4.0 with $S_{1}$ period showed a different variation pattern from that by GIPSY. Semi-diurnal oscillation mode appeared in dPWV by Bernese Ver. 4.0. In the double difference method, a miss modeling of the site displacement at one site affected solutions at other sites. There is a possibility that the error at one of the reference sites, where the OTL is large, had an influence on the ZTD analysis at MRI. However, there also remains the possibility that composite data did not sufficiently eliminate other irrelevant components because the data was retrieved at hourly intervals.

Acknowledgments. The authors would like to thank referees for their variable comments which enabled us to improve the manuscript, Tadahiro Sato (National Astronomical Observatory of Mizusawa) and Arata Sengoku (Hydrographic Department, Japan Coast Guard) for providing us with GOTIC software and site displacement sequence calculation utility, and Caltech/JPL for the GIPSY/OASIS II package. This research project is part of "GPS Meteorology Project in Japan", which is financially supported by the Japanese Science and Technology Agency.

\section{References}

Beutler, G., I. Bauersima, W. Gurtner, M. Rothacher, T. Schildknecht, and A. Geiger, Atmospheric refraction and other important biases in GPS carrier phase observation, in Atmospheric Effects on Geodetic Measurements, Monograph, 12, pp. 15-43, School of Surveying, Univ. of New South Wales, Kensington, Australia, 1988.

Bevis, M., S. Businger, T. A. Herring, C. Rocken, R. A. Anthes, and R. H. Ware, GPS meteorology: Remote sensing of atmospheric water vapor using the Global Positioning System, J. Geophys. Res., 97, 15787-15801, 1992.

Davis, J. L., T. A. Herring, I. I. Shapiro, A. E. E. Rodgers, and G. Elgered, Geodesy by radio interferometry: Effects of atmospheric modeling errors on estimates of baseline length, Radio Sci., 20, 1593-1607, 1985.

Duan, J., M. Bevis, P. Fang, Y. Bock, S. Chiswell, S. Businger, C. Rocken, F. Solheim, T. VonHove, R. Ware, S. McClusky, T. A. Herring, and R W. King, GPS meteorology: Direct estimation of the absolute value of precipitable water, J. Appl. Meteor, 35, 830-838, 1996

McCarthy, D. D., IERS Standards (1992), IERS Technical Note 13, 1992.

Niell, A. E., Global mapping functions for the atmospheric delay at radio wavelengths, J. Geophys. Res., 101(B2), 3227-3246, 1996.

Ohtani, R. and I. Naito, Comparison of GPS derived precipitable water vapors with radiosonde observation in Japan, J. Geophys. Res., 2000 (in press).

Rothachar, M. and L. Mervart, The Bernese GPS software version 4.0 user manual report, Astron. Inst., Univ. of Bern, Bern, Switzerland, 1996.

Sato, T. and H. Hanada, A Program for the Computation of Oceanic Tidal Loading Effects 'GOTIC', Publications of the International Latitude Observatory of Mizusawa 18, 29-47, 1984.

Schwiderski, E. W., NSWC Global Ocean Tide Data (GOTD) Tape, U. S. Naval Surface Warfare Center, Dahlgren, Virginia, 1981.

Sengoku, A. and T. Sato, Site displacement due to Solid Earth Tide and Ocean Loading at SLR, VLBI and GPS Observation Sites in Japan, Journal of the Japan Society for Marine Surveys and Technology, 7, 1-7, 1995 (in Japanese).

Sengoku, A. and T. Sato, Ocean loading site displacement around Japan, in Report of Hydrographic Researches, 32, pp. 129-135, Hydro. Depart., Tokyo, 1996 (in Japanese).

Webb, F. H. and J. F. Zumberge, An introduction to the GIPSY/OASIS-II, JPL Publ., D-11088, 1993.

Williams, J. G., Solid Earth Tides, IOM 391-109, Jet Propulsion Laboratory, Pasadena, Cal., 1970.

Y. Shoji (e-mail: yshoji@mri-jma.go.jp), H. Nakamura, K. Aonashi, A Ichiki, H. Seko, and Members of GPS/MET Japan Summer Campaign 1997 in Tsukuba 